\title{
MUERTES EN LAS CÁRCELES URUGUAYAS MAGNITUD DEL FENÓMENO Y PROBLEMAS PARA ESTUDIARLO
}

\author{
Ana Vigna y Santiago Sosa Barón
}

\begin{abstract}
Resumen
El sistema penitenciario destaca como un espacio en el cual la conflictividad y la violencia se vuelven muy evidentes. Las defunciones en este contexto cobran rasgos específicos, tanto en términos de incidencia de los distintos tipos de muerte como en los perfiles de la población fallecida. A pesar de la relevancia y la magnitud del problema, existen grandes dificultades para estudiarlo de manera empírica, vinculados - entre otras cosas - a la calidad de la información disponible $y$ al acceso a ella. Este artículo tiene un objetivo triple: reflexionar acerca de las fuentes de datos disponibles en Uruguay para el análisis del fenómeno; proveer un panorama general de las muertes ocurridas en las cárceles uruguayas en la última década; y enumerar algunos de los principales desafíos que tiene el país para enfrentar esta problemática.
\end{abstract}

Palabras clave: muertes bajo custodia, violencia, cárcel.

\begin{abstract}
Deaths in Uruguayan prisons. Magnitude of the phenomenon and problems for its study The penitentiary system stands out as a space in which conflict and violence becomes particularly evident. Deaths in this context acquire specific features, either in terms of incidence of the different types of death, or in the profiles of the deceased population. Despite the relevance and magnitude of the problem, there are strong difficulties for its empirical study, linked -among other thingsto the access and quality of available information. The article has a threefold objective: to reflect on the availability of data sources for the analysis of the phenomenon; to provide a general overview of the deaths that have taken place in Uruguayan prisons in the last decade; and to enumerate some of the main challenges that the country faces to address this problem.
\end{abstract}

Keywords: deaths in custody, violence, prisons.

Ana Vigna: Docente e investigadora del Departamento de Sociología, Facultad de Ciencias Sociales, Universidad de la República (UdelaR, Uruguay). Integrante del Núcleo de Análisis de la Criminalidad y la Violencia. Candidata a doctora en Sociología por la UdelaR y la Universidade de São Paulo (Brasil).

ORCID iD: https://orcid.org/0000-0003-2312-0913

E-mail: ana.vigna@cienciassociales.edu.uy

Santiago Sosa: Licenciado en Sociología, Facultad de Ciencias Sociales, UdelaR. Estudiante de la Maestría en Ciencias Humanas opción Filosofía Contemporánea y del Diploma en Políticas Públicas en Crimen e Inseguridad (UdelaR). Responsable de estadísticas y análisis de la oficina del Comisionado Parlamentario para el Sistema Penitenciario.

ORCID iD: https://orcid.org/0000-0002-7157-0946

E-mail: sosabaron@gmail.com

Recibido: 6 de junio de 2018.

Aprobado: 6 de octubre de 2018. 


\section{Introducción}

Uruguay es un país que se destaca en el contexto regional por su calidad democrática, por los avances observados en los últimos años en relación con la nueva agenda de derechos ${ }^{1}$ y por su buena posición en lo que refiere a diversos indicadores de desarrollo y calidad de vida. ${ }^{2}$ En relación con el tratamiento del delito, en 1907 quedó abolida la pena de muerte, mientras que el artículo 26 de la Constitución de la República indica que: "En ningún caso se permitirá que las cárceles sirvan para mortificar, y sí solo para asegurar a los procesados y penados, persiguiendo su reeducación, la aptitud para el trabajo y la profilaxis del delito".

A pesar de estos rasgos, que posicionan a Uruguay en un lugar privilegiado en el imaginario social, el país muestra un carácter fuertemente punitivo en el trato que les destina a las personas que han delinquido. Así, y si bien tiene cifras relativamente bajas para el contexto regional en materia de violencia y criminalidad, Uruguay presenta la segunda tasa más alta de encarcelamiento de Sudamérica (International Centre for Prison Studies, 2018). Sin embargo, la punitividad de un país no se mide solo por la proporción de ciudadanos que tiene viviendo tras las rejas, sino también por el trato que les brinda durante el período de reclusión. Así como la tasa de homicidios es a menudo utilizada como indicador para comparar los niveles de criminalidad de los países, las muertes bajo custodia penitenciaria resultan un buen indicador para medir no solo los diferentes grados de "aceptabilidad" que las sociedades tienen ante al sufrimiento de la población privada de libertad, sino también para evaluar la calidad de vida en los establecimientos de reclusión.

En este sentido, la muerte de los ciudadanos, y en particular la muerte violenta, constituye un hecho de alta gravedad. Pero cuando estos decesos se dan dentro del marco del sistema penitenciario, donde el Estado debe actuar como garante de los derechos de las personas recluidas, la situación adquiere un estatus especial. El hecho de que algunas cárceles sean más "sobrevivibles" que otras (Liebling, 2011) nos hace reflexionar, por un lado, sobre que la situación de encierro siempre conlleva una serie de sufrimientos o dolores que trascienden la privación de la libertad (Sykes, 1958; Goffman,

1 Entre las conquistas más salientes se encuentran la regulación del mercado de cannabis, la aprobación del matrimonio igualitario y la despenalización del aborto, entre otras.

2 Así, por ejemplo, el Índice de Desarrollo Humano del país ubica a Uruguay en el tercer lugar en América Latina en 2018 (Programa de Naciones Unidas para el Desarrollo-Uruguay, 2018). 
2001[1961]). Por otro lado, da cuenta de la gran heterogeneidad que existe entre los distintos sistemas penitenciarios, pero también, dentro de cada sistema, entre los diferentes establecimientos de reclusión.

Por su parte, la muerte bajo custodia penitenciaria no puede pensarse como un fenómeno homogéneo, sino que presenta una gran variabilidad y deriva de procesos causales diversos. Grosso modo, pueden identificarse tres grandes tipos de muertes: los suicidios, los homicidios y las muertes naturales.

En relación con el suicidio, Dooley (1990a) desarrolla un estudio sobre el fenómeno en las cárceles de Inglaterra y Gales y encuentra que entre los perfiles de los suicidas están sobrerrepresentados quienes tienen antecedentes de tratamiento psiquiátrico y de autolesiones. Por su parte, Liebling (2011) destaca que las condenas largas e indeterminadas, la escasa capacidad para desarrollar actividades y la desconfianza institucional incrementan la sensación de desesperanza por parte de los internos. El aislamiento con respecto al exterior, la incertidumbre cotidiana y la falta de control sobre la propia vida han sido identificados como elementos clave en la intensificación del estrés y la angustia, sobre todo en las personas más vulnerables (Liebling, 1999). Las dificultades de acceso a los servicios de salud y, en particular, a los de salud mental, los altos niveles de conflictividad y violencia, las dificultades en el acceso a la comida y a la vestimenta y los problemas derivados de la convivencia forzada hacen que, a menudo, la privación de libertad se transforme en "privación de significado y de biografía" (Liebling, 2011).

En cuanto al homicidio, Liebling (2017) menciona que hay elementos vinculados al mundo del delito y a la propia cultura carcelaria (deudas derivadas del tráfico de drogas, el tipo de delito cometido, enemistades previas al encarcelamiento o relativas al mantenimiento del estatus y el poder en la prisión) que podrían actuar como disparadores de este tipo de incidentes. Sin embargo, observamos que muchos homicidios ocurridos en cárceles fueron desatados por cuestiones aparentemente menores, vinculadas a la propia convivencia dentro de la prisión. La superpoblación y la falta de personal son dos elementos clave en la proliferación de la violencia letal (Liebling, 1999) e indican grados variables de negligencia institucional.

Por su parte, la calificación de una porción de las muertes ocurridas en prisión como "naturales" resulta muy problemática, ya que, retomando un informe del Comisionado Parlamentario para el Sistema Penitenciario, se supone que la muerte natural debería referir a:

"[...] causas exclusivamente endógenas al organismo, que tuvieron que ver con enfermedades que desembocaron en la muerte de la persona. El concepto presupone así, que en todos estos casos, el tratamiento de salud por parte de la institución fue óptimo y que no hubo aspectos exógenos, "no naturales", estructurales o contingentes de las condiciones de reclusión y de la atención de salud en los centros, que pudieron haber evitado o 
influido en dichas muertes." (Comisionado Parlamentario para el Sistema Penitenciario, 2016, p. 5)

Sin embargo, y conociendo las malas condiciones de vida y las dificultades en el acceso a los servicios de salud que presenta gran parte de las personas privadas de libertad en el contexto uruguayo, esta calificación resulta, por lo pronto, cuestionable. ${ }^{3}$

Las teorías explicativas de la violencia carcelaria se centran en dos grandes grupos de factores. Por un lado, la perspectiva de la importación (Irvwin y Cressey, 1962; Dawes, 1999) hace énfasis en los factores individuales y entiende que la mayor incidencia de la violencia en las prisiones en comparación con el mundo extramuros puede explicarse porque en las unidades carcelarias están sobrerrepresentadas las personas que tienen cierta predisposición a recurrir a la violencia como modo de resolver los conflictos, quienes tienen dolencias psiquiátricas y quienes no disponen de los recursos necesarios para lidiar con los diversos problemas que enfrentan. Así, desde esta perspectiva se enfatizan aquellos elementos que ya traen consigo los individuos que acaban en prisión y que los hacen más proclives a ejercer comportamientos violentos o a ser víctimas de ellos. Por su parte, las teorías de la deprivación remarcan que son los elementos derivados de las propias condiciones del encierro o de la gestión de los establecimientos los que atentan contra la dignidad y la integridad de la vida (Sykes, 1958). ${ }^{4}$

Tomando en consideración ambas perspectivas, Liebling (2017) remarca que los factores individuales no solo no disminuyen la responsabilidad del Estado en relación con estas muertes, sino que ponen de manifiesto el deber que este asume al privar de su libertad a estas poblaciones y la consecuente necesidad de contar con equipos y recursos adecuados y suficientes como para garantizar la integridad física de las personas que viven bajo su tutela.

Con el objetivo de visibilizar el rol (activo o pasivo) del Estado en las muertes ocurridas bajo custodia penitenciaria, el Mecanismo Nacional de Prevención de la Tortura de Paraguay (2017) realiza una clasificación de los fallecimientos según la categoría de responsabilidad estatal, distinguiendo entre muertes debidas a: I) la omisión del Estado en la posición de garante del derecho a la salud; II) la omisión del Estado en la posición de garante en las medidas de seguridad preventiva de la violencia entre personas privadas de libertad; III) la omisión del Estado en la posición de garante en la prevención de siniestros; IV) el uso de la fuerza letal por parte de agentes penitenciarios; V) la omisión del Estado en la posición de garante ante situaciones de huelga de hambre; y VI) la omisión del Estado en la posición de garante ante suicidios.

3 Para profundizar en los límites y las implicancias del concepto de "muerte natural" en el ámbito penitenciario, ver Sosa Barón (2018).

4 Para obtener más detalles sobre las discrepancias entre la teoría de la importación y la teoría de la deprivación, ver Trajtenberg y Sánchez de Ribera (2019) en este mismo número. 
En Uruguay, el tema de las muertes bajo custodia apenas ha comenzado a señalarse en el debate público, quizás vinculado a una nueva sensibilidad frente a los niveles de violencia en prisiones. ${ }^{5}$ Aun así, la bibliografía específica es muy incipiente. La principal referencia al respecto es el Informe especial sobre muertes en prisión en 2016, del Comisionado Parlamentario para el Sistema Penitenciario (2016), en el que por primera vez se publicaron estadísticas sobre los fallecimientos en custodia durante los últimos años, junto con algunas líneas interpretativas. Así, de manera creciente se comenzó a alertar sobre la gravedad de los altos índices de muertes violentas (Mecanismo Nacional de Prevención de la Tortura-Uruguay, 2017) y a ofrecer explicaciones tentativas que apuntan a las malas condiciones de reclusión y a la ausencia de programas de rehabilitación (Talvi, 2017).

Si bien no hay registros sistemáticos de incidentes violentos o heridos en prisiones, un estudio epidemiológico de las condiciones de reclusión señala una alta incidencia de lesiones graves y de personas internadas a raíz de incidentes violentos, así como una presencia significativa de factores de riesgo de suicidio en la población penitenciaria (Levcovitz, et al., 2016). Por su parte, el tema de los suicidios en custodia también ha sido abordado desde la metodología de "autopsia psicológica", que explora en profundidad los factores personales, familiares y ambientales que afectan la salud mental de las personas privadas de libertad, a partir del estudio y el seguimiento de un caso de suicidio y con énfasis en las posibilidades del trabajo institucional para su prevención (Rossi, 2017).

Antes de pasar al siguiente apartado, resulta necesario destacar que no solo no existe consenso sobre los factores explicativos y la tipificación de este fenómeno, sino que la propia definición de muerte bajo custodia está sujeta a debate: ¿Hasta dónde se extiende este concepto? ¿Debería tomar en consideración tanto al sistema penitenciario adulto como al adolescente? ¿Y a los hospitales psiquiátricos y hogares de niños? ¿Qué sucede con las muertes ocurridas durante las salidas transitorias, en prisión domiciliaria o en hospitalizaciones luego de un traslado desde un centro de reclusión? La delimitación del concepto de custodia o incluso de custodia penitenciaria es objeto de discusión (Sánchez, 2016). ${ }^{6}$ Dentro de este campo de análisis de límites difusos, es necesario aclarar que en este artículo restringiremos el análisis estrictamente a las muertes

$5 \quad$ Arbesún señala cómo la naturalización de las muertes en prisiones podría explicar la pobreza histórica de los registros del fenómeno (2017, p. 179).

6 Para incrementar los niveles de comparabilidad internacional, el Council of Europe Annual Penal Statistics (Aebi, et al., 2017) incluye en las tablas en las que presenta los datos correspondientes a las muertes totales y a los suicidios ocurridos en las cárceles europeas información acerca de si cada país reporta: I) datos sobre los reclusos que murieron o cometieron suicidio en hospitales comunitarios y II) datos sobre los reclusos que murieron o cometieron suicidio fuera de la prisión (durante salidas transitorias o similares). 
ocurridas en cárceles de adultos, en función de que es el ámbito para el que se dispone de información.

El artículo se estructura del siguiente modo: luego de esta breve introducción, se presentan las fuentes de datos sobre el tema disponibles en Uruguay y se indican sus potencialidades y limitaciones. A continuación, se brinda un panorama general de las muertes bajo custodia penitenciaria ocurridas en el país: distribución, características y evolución en los últimos años, así como una comparación con el volumen y rasgos de las muertes extramuros. Para finalizar, se concluye con algunas reflexiones respecto de los principales desafíos que enfrenta el país en la materia.

\section{¿Qué sabemos de quienes mueren en las cárceles uruguayas?}

Antes del análisis de cuántas y quiénes son las personas que mueren en el sistema carcelario uruguayo, es necesario reflexionar sobre las fuentes de datos de las que disponemos para analizar este fenómeno. En Uruguay, el principal organismo productor de información sobre las muertes bajo custodia penitenciaria es el propio Instituto Nacional de Rehabilitación del Ministerio del Interior (INR-MI). El que las estadísticas oficiales sean la principal fuente de datos genera fuertes controversias respecto de su validez y confiabilidad. Aun en los países donde las condiciones de reclusión son muy superiores a las latinoamericanas, se destaca que la falta de capacitación específica para relevar y registrar la información y las prioridades laborales que tiene el personal penitenciario hacen que los datos resultantes sean de dudosa calidad (Liebling, 1999, p. 290), lo que deriva en múltiples errores y en la incompletitud de los registros. Estas limitaciones se ven acentuadas en el caso uruguayo, donde a los problemas de registro propios de la escasez de recursos humanos y técnicos idóneos se suman los conflictos de interés derivados de que el organismo estatal encargado de generar la información sobre las muertes bajo custodia es el mismo que actúa como responsable de la integridad física de las personas privadas de libertad.

Las limitaciones relativas a la calidad y la disponibilidad de la información se observan incluso en los datos más elementales para caracterizar a las muertes (tales como la identificación de la persona fallecida, el momento y el lugar del hecho y las causas del deceso). Sin embargo, Liebling (1999) destaca que para poder comprender el fenómeno no basta con tomar en consideración variables fácilmente cuantificables (tiempo de encarcelamiento, nivel de superpoblación o razón de internos por funcionario), sino que deberían relevarse también elementos relativos a los aspectos relacionales y vinculares de la vida en prisión (en particular, el vínculo entre internos y de estos con los funcionarios).

Lamentablemente, la situación en Uruguay respecto a la accesibilidad y la transparencia de la información sobre la realidad carcelaria dista mucho 
de ser la ideal. Al día de hoy no se cuenta con un sistema de monitoreo de los indicadores más elementales ni de su evolución. ${ }^{7}$ A diferencia de otros países de la región, como Argentina, Brasil o Chile, el servicio penitenciario uruguayo no publica estadísticas. En este contexto de opacidad, las muertes bajo custodia penitenciaria no representan una excepción. Por el contrario, Uruguay registra los menores avances en la región (Gual, 2016, p. 33), situación potenciada hasta 2016 por la inexistencia del seguimiento de este tema en "informes sombra" de organizaciones de derechos humanos (Gual, 2016, p. 37).

A partir de 2016, el equipo del Comisionado Parlamentario para el Sistema Penitenciario comenzó a relevar y registrar información básica de cada una de las muertes ocurridas en cárceles, dando inicio al seguimiento sistemático del tema. Ante la carencia de datos públicos para años anteriores, la oficina realizó un pedido de información al Instituto Nacional de Rehabilitación, que permitió reconstruir la serie de $2006^{8}$ a 2015 en relación con la cantidad de muertes por establecimiento, según año de ocurrencia y tipo. Para los sucesos posteriores a 2016, el Comisionado inició un relevamiento continuo de información mediante un formulario en el que se recogían datos básicos sobre la persona fallecida (nombre, edad, fecha de fallecimiento, situación procesal, carátula y antecedentes) y la causa de la muerte, y un breve relato del hecho. También se intentó relevar información sobre la historia personal del fallecido, su familia, sus actividades dentro de la prisión y sus evaluaciones y planes de tratamiento dentro de la prisión. Sin embargo, al depender el llenado de esta ficha de la escasa información disponible en los registros oficiales, tuvo pocos resultados en estas dimensiones.

Desde entonces el tema comenzó a estar en la agenda de comunicación entre la oficina del Comisionado Parlamentario y el Instituto Nacional de Rehabilitación, y durante 2016 y 2017 el Comisionado fue notificado de manera informal de las muertes ocurridas en el sistema penitenciario (en general, el mismo día) o tomó conocimiento a través de las visitas regulares de monitoreo que realiza a las cárceles del país. Si bien este mecanismo representó una mejora sustantiva en relación con la información disponible para años anteriores y se redujo sensiblemente el riesgo de cifra negra, debe destacarse que las muertes por enfermedad no siempre fueron notificadas en fecha y en algunos casos no fueron notificaron. A fines de 2017, se cotejaron los registros con el Ministerio del Interior y se corroboró una cifra total en común.

7 En muchos casos la información existe pero es muy precaria, con dificultades en el registro sistemático de eventos. En su Informe Anual 2017, el Comisionado Parlamentario para el Sistema Penitenciario señala: "[...] todavía hay importantes carencias para recoger la información, contar con indicadores significativos y hacer accesible la información a todos los actores para tomar decisiones [...]" (2018, p. 81).

No existen datos sistematizados de muertes en custodia anteriores a 2006. 
A partir de octubre de 2017, además de la notificación informal y del conocimiento a través del monitoreo habitual, el Ministerio del Interior comenzó a enviar de modo sistemático (cada uno o dos meses) a la oficina del Comisionado un listado de las muertes ocurridas durante el período. Gracias a este proceso, las diferencias en la contabilización de los decesos han sido cada vez menores $y$, por lo general, refieren a muertes por enfermedad que no fueron comunicadas en fecha. El listado de muertes que envía el Ministerio contiene información relativa a: I) la fecha del fallecimiento; II) el nombre del fallecido; III) la unidad de alojamiento; y IV) la causa del fallecimiento (según la categorización: homicidio, suicidio, accidente, muerte natural). ${ }^{9}$

En 2017, con base en los resultados del relevamiento anterior y en el intercambio con la Procuración Penitenciaria de la Nación (Argentina), el Comisionado Parlamentario actualizó su formulario de relevamiento y mejoró el protocolo de seguimiento. ${ }^{10}$ La nueva ficha incluye una clasificación de tipos de muerte y otra de causas de muerte, asimiladas a las que utiliza la Procuración en las cárceles federales argentinas, que permiten definir con mayor precisión los fallecimientos. También incluye un módulo especial para los suicidios, en el que se indaga sobre antecedentes de intentos de autoeliminación, ideación suicida, si el fallecido compartía celda con otras personas y el estado de ánimo previo al suicidio. Asimismo, se incorporó un módulo para las muertes por enfermedad, en el que se registran los detalles de la historia clínica. Se releva, además, la fecha de comienzo de la privación de libertad y la fecha de ingreso a la última unidad. Pese a las mejoras en el instrumento de relevamiento, resta evaluar si se obtienen mejores resultados en los aspectos contextuales del episodio del fallecimiento y la información básica de la persona. Dado que las carencias de información en las carpetas de los internos aún son notorias, el relevamiento planificado demandaría una movilización de recursos para investigar las muertes (averiguaciones en las oficinas técnicas y jurídicas, en los servicios de salud, con los compañeros de celda, con los familiares, etcétera) con los que la oficina del Comisionado no cuenta.

Finalmente, cabe destacar que el Ministerio del Interior, a través del Sistema de Gestión de Seguridad Pública, releva información relativa a la edad y el sexo de todas las víctimas de suicidio y homicidio del país. En 2018, a partir de un pedido realizado por el Departamento de Sociología de la Facultad de Ciencias Sociales de la Universidad de la República, el Ministerio del Interior brindó información de todas las víctimas de homicidios para el período 2013-2017 y de los suicidios ocurridos en el país en los años 2016-

9 Cabe destacar que este envío incluye también una lista similar para las muertes ocurridas en salidas transitorias y para las muertes en prisión domiciliaria.

10 Para conocer más sobre el procedimiento administrativo activado por la Procuración Penitenciaria de Argentina cada vez que se produce una muerte bajo custodia penitenciaria, ver Gual (2019) en este mismo número. 
2017, distinguiendo si ocurrieron dentro o fuera de los establecimientos de reclusión. En la Tabla 1 se sintetizan las dimensiones relevadas por cada una de las fuentes de información disponibles.

\section{Tabla 1. Resumen de fuentes de información y dimensiones de registro de muertes en custodia}

\begin{tabular}{|c|c|c|}
\hline Período & Institución & Dimensiones \\
\hline 2006-2016 & $\begin{array}{l}\text { Instituto Nacional de } \\
\text { Rehabilitación-Ministerio del } \\
\text { Interior }\end{array}$ & Tipo de muerte, año, unidad penitenciaria \\
\hline 2016 & $\begin{array}{l}\text { Instituto Nacional de } \\
\text { Rehabilitación-Ministerio del } \\
\text { Interior }\end{array}$ & $\begin{array}{l}\text { Fecha, unidad penitenciaria, nombre, delito, } \\
\text { edad, antecedentes penales, causa de } \\
\text { muerte (se registran también las muertes en } \\
\text { salidas transitorias y en prisión domiciliaria) }\end{array}$ \\
\hline 2013-2017 & $\begin{array}{l}\text { Sistema de Gestión de } \\
\text { Seguridad Pública e Instituto } \\
\text { Nacional de Rehabilitación- } \\
\text { Ministerio del Interior }\end{array}$ & $\begin{array}{l}\text { Año, edad y sexo de las víctimas de } \\
\text { homicidios }\end{array}$ \\
\hline 2016-2017 & $\begin{array}{l}\text { Sistema de Gestión de } \\
\text { Seguridad Pública e Instituto } \\
\text { Nacional de Rehabilitación- } \\
\text { Ministerio del Interior }\end{array}$ & Año, edad y sexo de las víctimas de suicidios \\
\hline 2016 & $\begin{array}{l}\text { Comisionado Parlamentario } \\
\text { para el Sistema Penitenciario }\end{array}$ & $\begin{array}{l}\text { Fecha, lugar del fallecimiento, datos } \\
\text { personales y jurídicos, causa de muerte, } \\
\text { información sobre actividades de la víctima } \\
\text { en prisión, breve relato del hecho }\end{array}$ \\
\hline 2017-2018 & $\begin{array}{l}\text { Comisionado Parlamentario } \\
\text { para el Sistema Penitenciario }\end{array}$ & $\begin{array}{l}\text { Ídem } 2016 \text {, módulo sobre suicidio, } \\
\text { información de la historia clínica en muertes } \\
\text { por enfermedad }\end{array}$ \\
\hline 2017-2018 & Ministerio del Interior & $\begin{array}{l}\text { Nombre, fecha, tipo de muerte, unidad } \\
\text { penitenciaria (se registran también las } \\
\text { muertes en salidas transitorias y en prisión } \\
\text { domiciliaria) }\end{array}$ \\
\hline
\end{tabular}

Fuente: Elaboración propia, 2018.

Como fue adelantado, uno de los aspectos particularmente sensibles en relación con este tema refiere a la clasificación de los tipos de muerte. A partir del avance realizado por la Procuración Penitenciaria, el equipo del Comisionado Parlamentario adoptó un esquema de clasificación que permite 
distinguir tipos de causas de muerte. Así, los tipos de muerte distinguen: 1) homicidio, 2) suicidio, 3) accidente, 4) causa dudosa (violenta), 5) enfermedad, 6) muerte súbita, 7) causa dudosa (no violenta), 8) violencia institucional. Por su parte, las causas de muerte registran si la muerte se derivó de: 1) ahorcamiento, 2) herida de arma blanca, 3) herida de arma de fuego, 4) incendio (quemadura, asfixia), 5) caída de altura, 6) asfixia con intervención de terceros, 7) otra causa violenta, 8) VIH (enfermedad oportunista), 9) otra enfermedad, 10) muerte súbita, 11) otra causa no violenta. Así, a partir de 2017 la categorización llevada adelante por el Comisionado Parlamentario es distinta a la del Ministerio del Interior, que continúa distinguiendo solo entre homicidios, suicidios, accidentes y muertes naturales.

Más allá de la mayor especificidad de la propuesta reciente, en la mayoría de los casos no hay discrepancias entre ambos registros. Sin embargo, pueden destacarse algunas excepciones significativas en las que se registraron versiones diferentes de los hechos y en algunos de estos casos directamente no hubo coincidencia en la clasificación. El Comisionado Parlamentario señaló que una de las tres muertes calificadas como accidente por el Ministerio del Interior en 2016 se había producido en el marco de un episodio de violencia interpersonal, ${ }^{11}$ mientras que en otra de ellas había indicios testimoniales de un posible suicidio. En 2017, una muerte calificada como accidente por el Ministerio del Interior fue, según el relevamiento del Comisionado, por una causa dudosa violenta que nunca fue aclarada. ${ }^{12}$ Por su parte, en 2018 hubo discrepancias en dos fallecimientos. Uno de ellos fue clasificado por el Ministerio como muerte natural y el Comisionado como causa dudosa violenta, mientras que el otro fue clasificado por el Ministerio como homicidio entre internos y el Comisionado lo registró como una muerte por violencia institucional, a raíz de un disparo de escopeta (Parlamento del Uruguay, 2018). Esta última discrepancia tuvo gran cobertura mediática y generó preocupación en la opinión pública cuando, varias semanas después de la muerte, trascendió que la versión difundida por el Ministerio era incorrecta. Estos casos no solo ponen de manifiesto el escaso detalle y la baja calidad de la información disponible sobre el fenómeno, sino que también dejan en evidencia las fuertes limitaciones relativas a las investigaciones de las muertes en custodia penitenciaria. En este sentido, la importancia de fortalecer los monitoreos independientes y la transparencia y la comunicación de los resultados de las pesquisas se vuelve más que evidente.

11 Con versiones contrapuestas respecto a si la caída de altura que desembocó en la muerte se había producido mientras recibía disparos de la guardia con munición no letal (Comisionado Parlamentario para el Sistema Penitenciario, 2017, p. 11).

12 En las actas aparecen versiones contradictorias sobre un posible suicidio u homicidio, aunque la muerte fue claramente violenta. 


\section{Panorama de las muertes en cárceles uruguayas}

Como se mencionaba antes, las muertes en prisión pueden ser entendidas como un indicador de las condiciones de reclusión de un determinado país en un momento dado. Para el caso uruguayo, deben ser comprendidas en un contexto en el que la cantidad de personas privadas de libertad ha aumentado de manera constante en las últimas décadas, casi cuadruplicándose desde 1990. Si bien se observó un leve detenimiento en torno al año 2005, cuando fue aprobada la Ley de Humanización Carcelaria, rápidamente fue revertido. El crecimiento sostenido continuó hasta 2017, año en el que se alcanzó un máximo histórico de 11.253 personas presas. A partir del segundo semestre de ese año, comenzó un leve descenso que se afirmó con la aprobación del nuevo Código del Proceso Penal. Este descenso se sostuvo algunos meses, hasta registrar 9.906 personas en junio de 2018, para luego retomar un proceso de crecimiento que continúa en la actualidad. La alta prisionización representa una fuerte presión sobre las posibilidades de gestión del sistema, y lleva a situaciones crónicas de hacinamiento, precariedad de las condiciones de vida, violencia y diversas violaciones de derechos humanos.

\section{Gráfico 1. Evolución anual de la tasa de prisionización de la población adulta privada de libertad (1988-2018]}

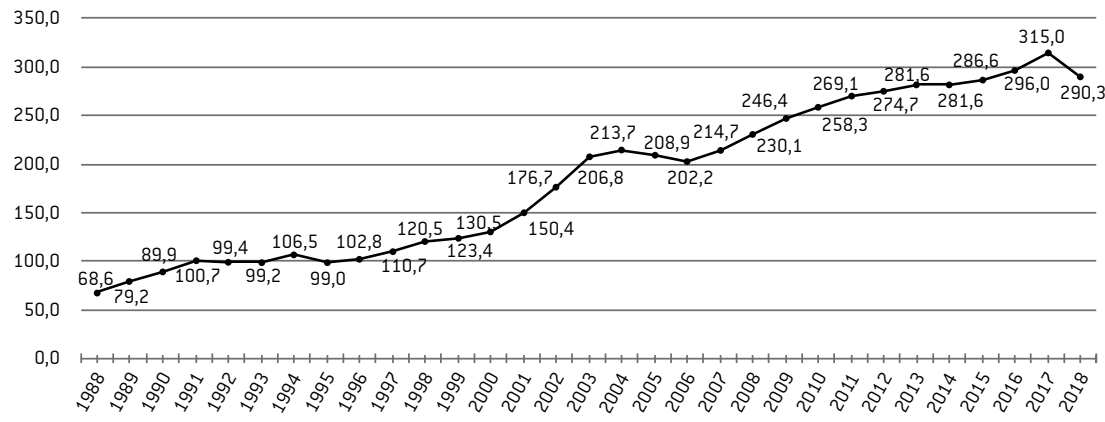

Fuente: Elaboración propia con base en datos del INR-MI.

A pesar de los grandes avances que se han observado en los últimos años a partir de la puesta en marcha de la reforma penitenciaria, la unificación del sistema en la órbita del Instituto Nacional de Rehabilitación, la incorporación de operadores penitenciarios y el fortalecimiento de la dimensión técnica de la intervención, en amplios sectores del sistema carcelario uruguayo las condiciones de vida continúan siendo alarmantes. Según el informe 2017 del Comisionado Parlamentario para el Sistema Penitenciario, tres de cada diez 
personas presas en Uruguay viven en condiciones de trato cruel, inhumano o degradante. ${ }^{13}$

En este marco, la muerte bajo custodia, lejos de mostrar una disminución significativa, en los últimos años ha permanecido constante. Si consideramos los doce años para los que se dispone de información (2006-2017), hubo en total 475 fallecimientos en cárceles, lo que da un promedio de alrededor de 40 personas privadas de libertad fallecidas al año. Si bien se observan algunos períodos de ascenso y descenso, el fenómeno ha adquirido un carácter endémico. En los últimos tres años (2015-2017) ocurrió el mismo número de muertes cada año: 47. Aunque existe una cifra importante de muertes evitables, en Uruguay, a diferencia de otros países de la región, no han tenido lugar grandes matanzas o episodios masivos de violencia letal, con la excepción de las doce muertes en el incendio de la cárcel de Rocha, ocurrido en 2010.

A pesar de ello, el alto volumen de muertes ocurridas bajo custodia sugiere que el sistema penitenciario resulta un contexto particularmente necrogénico, generador de situaciones que desembocan en la muerte. Esto puede observarse a través de la comparación de los niveles de mortalidad dentro y fuera de la cárcel. Si bien este tipo de comparaciones resulta de utilidad por su sencillez para transmitir que el mundo carcelario tiene mayores niveles de letalidad que el extracarcelario, podría ser cuestionado desde el punto de vista metodológico debido al fuerte impacto que tiene el cambio, de un año a otro, en algunas pocas unidades en el numerador (cantidad de muertes) cuando se trata de un denominador relativamente pequeño (cantidad de personas presas). A los efectos de lograr una comparación que implique más estabilidad en la medida, procedimos a calcular las tasas de homicidio y de suicidio tomando todo el período para el cual se dispone de información.

Estos cálculos nos indican que la tasa de homicidio en prisiones para el período 2006-2017 fue de 11,7 cada 10.000 internos (117 cada 100.000), cifra que es más de 16 veces mayor que la tasa de homicidios nacional promedio (7 cada 100.000), para el mismo período. Algo similar puede observarse respecto a los suicidios, que en custodia promediaron durante el período 7,6 cada 10.000 internos (75,9 cada 100.000), mientras que la tasa nacional promedio de suicidios para el período 2006-2017 fue de 17,4 cada 100.000 habitantes, según datos de la División de Estadísticas Vitales del Ministerio de Salud Pública (2017). Estas cifras reflejan que, más allá de los incrementos o descensos puntuales de la población privada de libertad y de las variaciones interanuales en la cantidad de homicidios o suicidios dentro de las cárceles,

13 En el mismo informe se estima que solo el $26 \%$ de la población se aloja en unidades donde hay oportunidades de integración social, mientras que el $44 \%$ restante se ubica en una categoría intermedia, donde hay "insuficientes condiciones para la integración social" (Comisionado Parlamentario para el Sistema Penitenciario, 2018a, p. 39). 
la prisión se presenta como un contexto donde la violencia interpersonal y las autoagresiones alcanzan volúmenes extremadamente elevados y mayores grados de gravedad, en relación con lo que ocurre en el mundo extramuros.

Además de los problemas estadísticos derivados de trabajar con denominadores pequeños, un cuestionamiento adicional que se le ha hecho a la comparación directa entre las cifras de muerte en el contexto penitenciario y en el mundo extramuros refiere a la estructura por sexo y edad de ambas poblaciones. Como es bien sabido, Uruguay cuenta con una población envejecida y que se distribuye de forma bastante equitativa entre hombres y mujeres. ${ }^{14}$ Por el contrario, la población penitenciaria está compuesta sobre todo por hombres jóvenes. Dado que la violencia letal, y en particular el homicidio, es protagonizada principalmente por varones jóvenes (tanto en el rol de víctimas como en el de victimarios), para comparar las cifras de violencia letal interpersonal ocurrida en la cárcel en relación con el resto de la sociedad, sería más preciso controlar los datos por sexo y edad.

Así, en el Gráfico 2 se presentan las tasas de homicidio por grupos de edades quinquenales exclusivamente para la población masculina. ${ }^{15}$ Se tomó en conjunto el período para el que se disponía de información (2013-2017) a los efectos de minimizar el impacto de variaciones coyunturales interanuales. A partir de los datos, se pueden realizar diversas observaciones. En primer lugar, resulta notorio el hecho de que la violencia letal interpersonal es un fenómeno que afecta en especial a la población masculina, en tanto la tasa de homicidios en los hombres es muy superior a la tasa nacional (que en los años referidos osciló entre 7,6 y 8,5 homicidios cada 100.000 habitantes) (Ministerio del Interior, 2018). En segundo lugar, se destaca que, aun controlando por edad y sexo, las tasas de homicidio intracarcelario superan ampliamente a las observadas fuera de la prisión, dado que son entre 3,5 y 9 veces superiores considerando a todos los grupos de edad entre 18 y 47 años. Por último, queda de manifiesto que la violencia letal es sufrida en particular por poblaciones juveniles, tanto dentro como fuera de la prisión. Sin embargo, mientras que en la vida extramuros los más jóvenes son quienes proporcionalmente se encuentran más expuestos a ser víctimas de violencia letal, dentro de la cárcel la tasa de homicidio afecta en términos proporcionales sobre todo al grupo de entre 33 y 37 años. Esto se debe a que en el sistema carcelario la población más joven se halla muy sobrerrepresentada (fenómeno que se acentúa aún más en el caso de los varones): el 58\% tiene hasta 30 años

14 Según datos del Instituto Nacional de Estadística (INE), en 2018 el 48,4\% de la población de Uruguay estaba conformado por hombres y el $51,6 \%$ restante por mujeres. Para ese mismo año, el índice de envejecimiento (cantidad de personas de 65 años y más por cada 100 personas menores de 15 años) era de 71,06 (Instituto Nacional de Estadística, 2018).

15 Se excluyó del análisis a la población femenina por presentar muy pocas observaciones durante el período analizado: entre 2013 y 2017 se registró un único homicidio en establecimientos carcelarios que tuvo como víctima a una mujer. 
y el $80 \%$ es menor de 40 años. ${ }^{16}$ Debido a ello, a medida que aumenta la edad el problema de los denominadores pequeños que mencionábamos antes se acentúa cada vez más, por lo que un cambio en una o dos muertes puede generar un fuerte impacto en la tasa de un grupo etario.

\section{Gráfico 2. Tasas de homicidio fuera y dentro de la cárcel por grupos de edades quinquenales, población masculina (cada 100.000 hombres). Período 2013- $2017^{17}$}

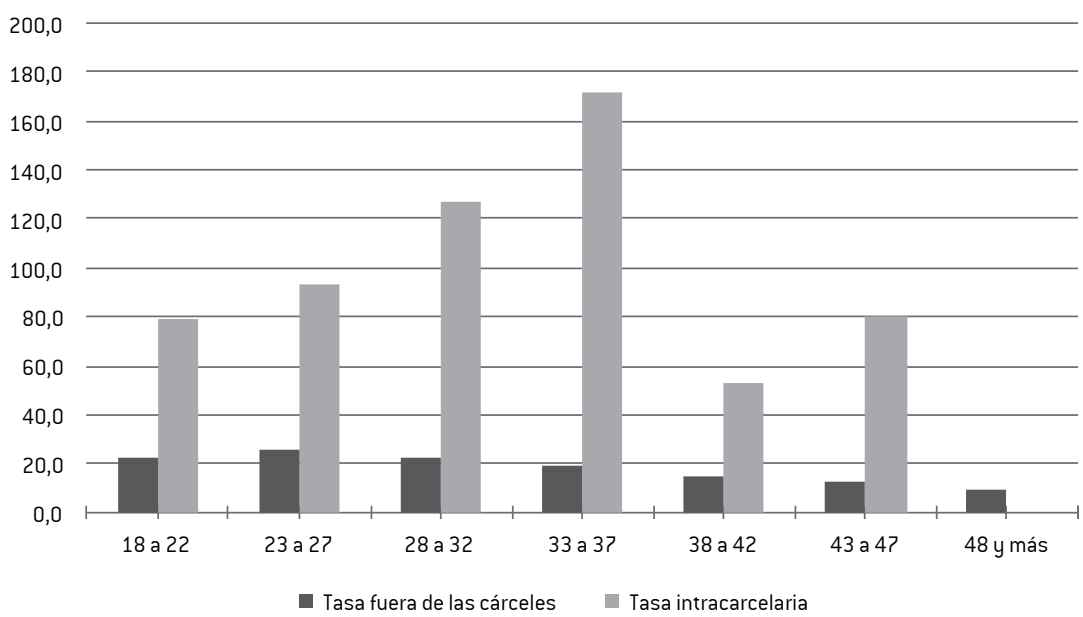

Fuente: Elaboración propia con base en datos del INE, el Sistema de Gestión de Seguridad Pública (MI) y el INR.

Otro indicador que ilustra el carácter necrogénico de la cárcel es la comparación de la edad promedio de muerte dentro y fuera del sistema penitenciario. En este sentido, llama poderosamente la atención el promedio de edad de las personas que mueren bajo custodia penitenciaria, que para Uruguay es de 37 años. Si consideramos solo las muertes violentas, el promedio es aún más bajo: 29 años para el caso de los homicidios (en comparación con los 35 años de las víctimas de homicidio extramuros) y

16 Estas cifras surgen de un procesamiento hecho por los autores de este artículo de la base de datos del Primer Censo Nacional de Reclusos, elaborado en 2010 por el Ministerio del Interior y el Departamento de Sociología de la Facultad de Ciencias Sociales de la Universidad de la República.

17 Para la elaboración de este gráfico se consideraron los datos aportados por el Ministerio del Interior sobre la estructura por sexo y edad de las víctimas de homicidio. Para el cálculo de las tasas extramuros se tomaron las estimaciones poblacionales del Instituto Nacional de Estadística. Ante la falta de datos actuales sobre la cantidad de personas privadas de libertad por edad, se procedió a tomar la distribución resultante del Primer Censo Nacional de Reclusos y estimar las proporciones acordes con el número de personas privadas de libertad por año. 
34 años para los suicidios (en comparación con los 47 años promedio de los suicidas fuera de las cárceles). Quienes mueren por otras causas violentas dentro de la prisión lo hacen en promedio a los 29 años. Por último, y aportando un nuevo elemento para el cuestionamiento de la etiqueta de "muerte natural", las personas que mueren en custodia por enfermedad lo hacen en promedio a los 48 años de edad.

Tabla 2. Edad promedio de las muertes dentro y fuera de las cárceles, según tipo [2016-2017]

\begin{tabular}{ccc}
\hline & \multicolumn{2}{c}{ Media de edad } \\
\hline Causa & Dentro de las cárceles* & Fuera de las cárceles \\
\hline Enfermedad/Natural & 48,3 & $\mathrm{~s} / \mathrm{d}$ \\
\hline Homicidio & 28,6 & 34,7 \\
\hline Suicidio & 33,9 & 46,5 \\
\hline Otras causas violentas & 29,5 & $\mathrm{~s} / \mathrm{d}$ \\
\hline Todas & 37,1 & $\mathrm{~s} / \mathrm{d}$
\end{tabular}

Fuente: Elaboración propia con base en relevamiento del Comisionado Parlamentario para el Sistema Penitenciario y el Sistema de Gestión de Seguridad Pública (MI).

* A partir de información de 92 de las 94 muertes ocurridas en el período. Se carece de información en dos muertes por enfermedad ocurridas en 2017.

Concentrándonos a partir de aquí solo en las muertes ocurridas dentro del sistema penitenciario, si se observa el período 2006-2017 de manera global, las muertes violentas (que incluyen los homicidios, suicidios, accidentes y otras causas violentas no del todo aclaradas) y las no violentas muestran una distribución muy similar (51\% y 49\% respectivamente). Sin embargo, al observar la distribución por año, se observan fuertes oscilaciones. En particular, llama la atención que en los últimos dos años las muertes violentas duplicaron a las no violentas. Al respecto, resulta pertinente recordar que a partir de 2016 las muertes tienen un monitoreo independiente a cargo de la oficina del Comisionado Parlamentario, con lo cual pudo haber mejorado la certidumbre de su clasificación. 
Gráfico 3. Distribución de muertes por año y tipo [2006-2017]

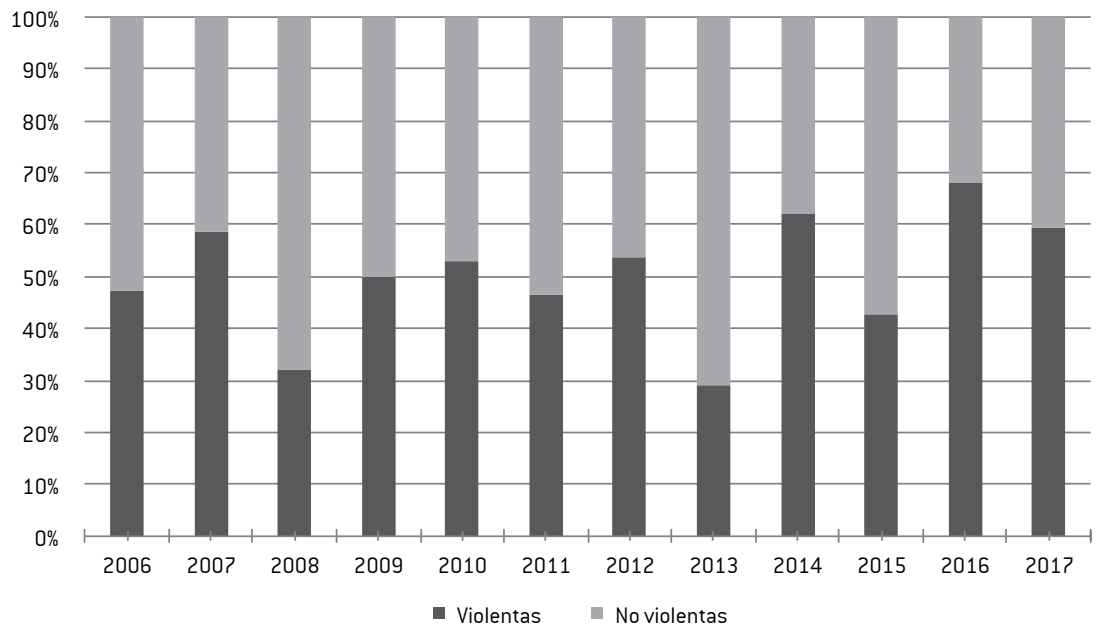

Fuente: Elaboración propia con base en datos del INR (proporcionados por el Comisionado Parlamentario para el Sistema Penitenciario].

El Gráfico 4 muestra la evolución anual de las muertes según tipo para el período 2006-2017, y permite observar una gran volatilidad. Como se mencionaba antes, las muertes no violentas constituyen la categoría modal, aunque en los años 2007, 2016 y 2017 su número es muy similar al de los homicidios. Por otro lado, las muertes violentas no aclaradas y los accidentes son los que reportan una menor incidencia durante todo el período, a excepción de 2010, cuando tuvo lugar el ya mencionado incendio en la cárcel de Rocha.

Si se considera el período global, de las 241 muertes violentas ocurridas a lo largo de los doce años para los cuales se tiene información, más de la mitad fueron homicidios (52\%), seguidos de suicidios (34\%) y, por último, muertes por accidentes o por otras causas violentas no del todo aclaradas $(14 \%)$. 


\section{Gráfico 4. Evolución anual de las muertes en custodia según tipo [2006-2017]}

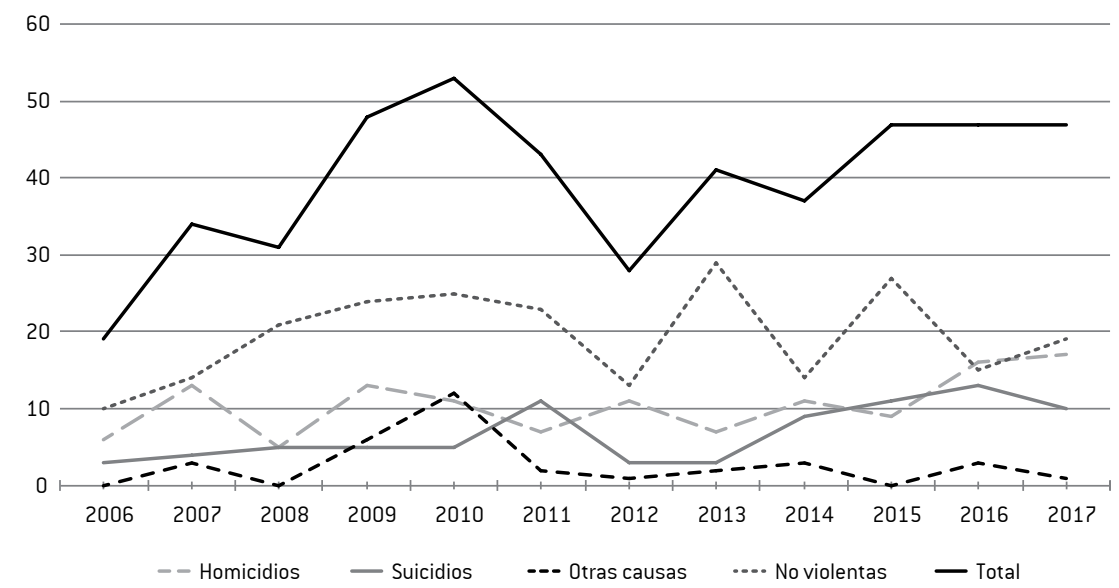

Fuente: Elaboración propia con base en datos del INR (proporcionados por el Comisionado Parlamentario para el Sistema Penitenciario].

El fenómeno de la muerte bajo custodia muestra gran heterogeneidad no solo según los distintos tipos de muerte, sino también según su distribución en el interior del sistema. En el Gráfico 5 se observa la distribución de los distintos tipos de muerte según unidad. Como es esperable, la enorme mayoría de las personas que fallecen en custodia lo hacen en las cárceles en donde se aloja la mayor proporción de personas. De este modo, más de dos tercios de las muertes han ocurrido en apenas dos unidades del área metropolitana: en la Unidad n. ${ }^{\circ} 4$ ex-Comcar (48\%) y en la Unidad n. ${ }^{\circ} 3$ Libertad (21\%). Estas cifras son similares si consideramos solo las muertes violentas: $41 \%$ en el ex-Comcar y $29 \%$ en Libertad.

Así, la Unidad n. ${ }^{\circ}$, que es el establecimiento de reclusión más poblado del país, concentra la mayor proporción de muertes de todos los tipos, excepto los suicidios. Estos últimos tienen una distribución más heterogénea y se concentran principalmente en la Unidad $n .^{\circ} 3$ (donde tienen lugar más de 3 de cada 10 suicidios), pero también encuentran una alta participación en otras unidades, como la n. ${ }^{\circ} 5$ (Femenino), la n. ${ }^{\circ} 13$ (Las Rosas, ubicada en el departamento de Maldonado) y otras del interior. En el ex-Comcar resulta llamativamente alta la proporción de muertes por enfermedad o "naturales" (un $56 \%$ del total). En la categoría "otras causas" destaca la Unidad n. ${ }^{\circ} 22$ Rocha, sobre todo a raíz del incendio. 


\section{Gráfico 5. Distribución de muertes por causa, según establecimiento [2006-} 2017]

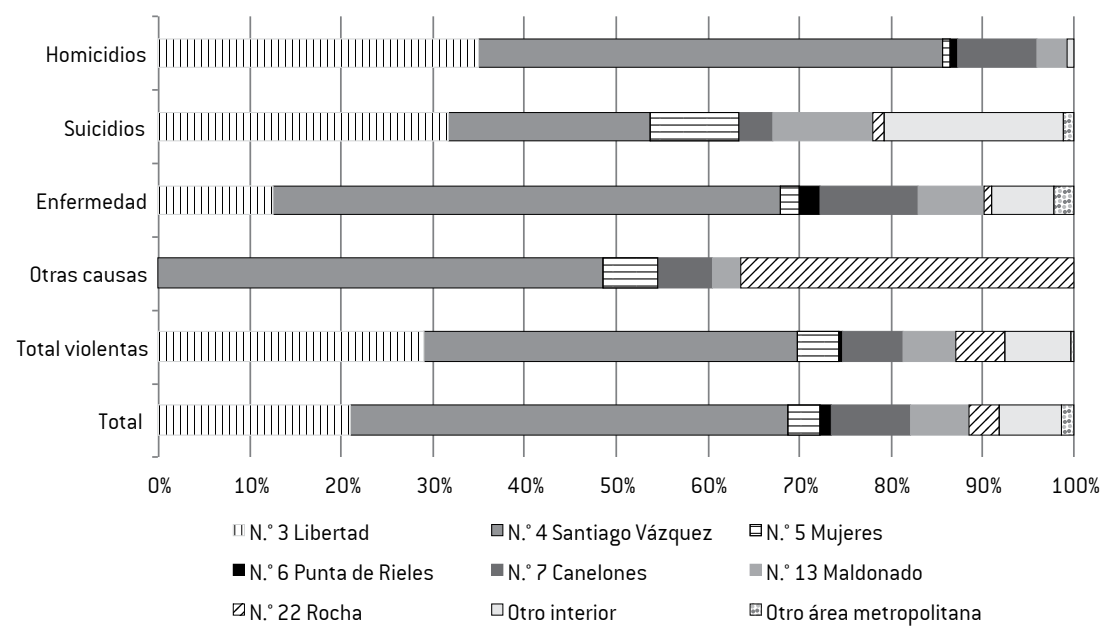

Fuente: Elaboración propia con base en datos del INR (proporcionados por el Comisionado Parlamentario para el Sistema Penitenciario].

Sin embargo, y dadas las grandes diferencias de población en las distintas unidades, es importante analizar la distribución de las muertes según la cantidad de internos. El Gráfico 6 presenta la cantidad de muertes ocurridas durante el período, en relación con la población promedio alojada entre 2006 y 2017 en las distintas unidades de internación. Así, aun controlando por cantidad de personas privadas de libertad, se observa que la muerte tiene una importante sobrerrepresentación en las grandes cárceles, como el ex-Comcar, Libertad, Canelones (Unidad n. $\left.{ }^{0} 7\right),{ }^{18} \mathrm{y}$ en la cárcel de mujeres. Pero además este análisis permite observar la sobrerrepresentación específica según los tipos de muerte, lo que redimensiona la incidencia de las muertes violentas en el Penal de Libertad, tanto respecto a los homicidios como a los suicidios.

Un hallazgo importante de este análisis es la gran incidencia de suicidios en la Unidad $\mathrm{n}^{\mathrm{0}} 5$, de mujeres. Como allí la población es bastante menor que en las grandes cárceles metropolitanas, la cantidad neta de suicidios puede

18 En estas grandes cárceles se observan ratios muy elevados de internos por funcionario y tiende a predominar una gestión despersonalizada, basada en el encierro, con una carencia crónica de programación de actividades y en la que se observan proporciones muy bajas de personas privadas de libertad que realizan actividades laborales y socioeducativas (Comisionado Parlamentario para el sistema penitenciario, 2017, pp. 63 y 71). También existe sobrepoblación respecto a las plazas disponibles, con sectores con hacinamiento muy crítico en ex-Comcar y Canelones (Comisionado Parlamentario para el Sistema Penitenciario, 2018b, pp. 13-14). Dado que no hay un criterio sistemático sólido de clasificación de la población privada de libertad, ni disponibilidad de información detallada en relación con sus perfiles, no es posible afirmar que exista una peculiaridad poblacional muy marcada en estas unidades que la diferencie de la del resto del sistema. 
pasar desapercibida. Sin embargo, si se observa la tasa, se manifiesta que es de las más altas del sistema, junto con la de la Unidad n. ${ }^{\circ} 3$ Libertad. Por último, este análisis permite destacar la altísima tasa de muertes catalogadas como "naturales" en el ex-Comcar, que afectan en promedio a 35 de cada 10.000 personas presas en dicho establecimiento.

\section{Gráfico 6. Tasas de muerte promedio [2006-2017) cada 10.000 personas privadas de libertad, por causa y establecimiento}

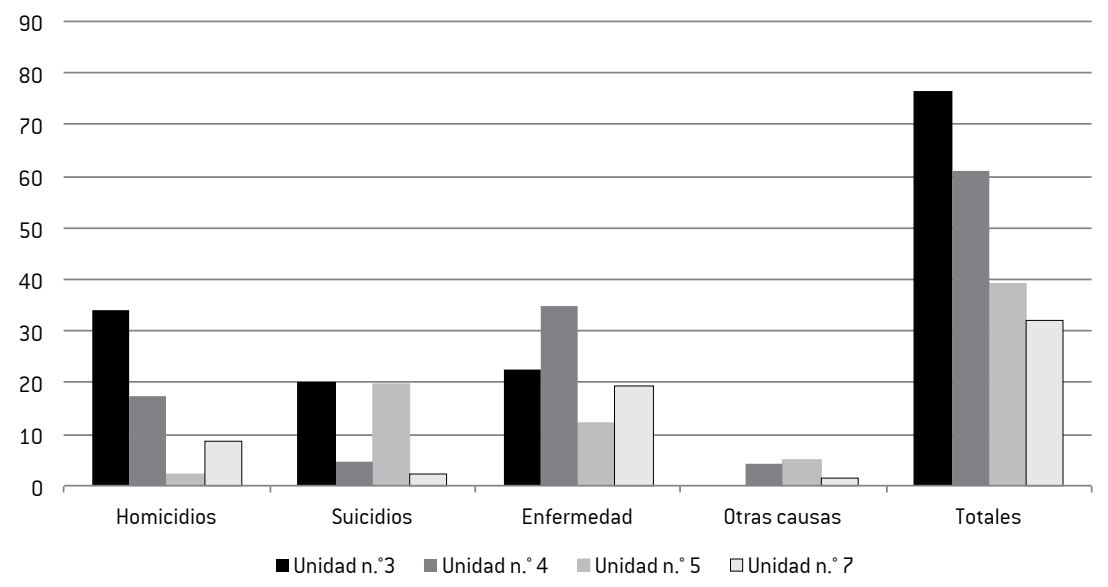

Fuente: Elaboración propia con base en datos del INR (proporcionados por el Comisionado Parlamentario para el Sistema Penitenciario) y el MI. Datos estimados de promedio poblacional para el año 2015 con base en información mayo-diciembre.

Para conocer mejor el fenómeno de la muerte bajo custodia penitenciaria, es necesario profundizar en las causas más próximas que suscitan los fallecimientos. Como se mencionó antes, a partir de 2017 el relevamiento continuo de la oficina del Comisionado Parlamentario comenzó a distinguir las causas de los tipos de muerte. A medida que el relevamiento avance y se acumulen más casos, el análisis de las modalidades y circunstancias de las muertes permitirá desarrollar políticas de prevención específicas, así como en el esclarecimiento de algunas incertidumbres sobre la participación de terceros o de eventuales responsabilidades estatales (Procuración Penitenciaria de la Nación, 2018, pp. 373-374), a las cuales la clasificación básica de tipo de muerte a menudo no es del todo sensible.

En la Tabla 3 se resumen las informaciones preliminares correspondientes a 2017. En uno de los fallecimientos (ocasionado por una caída de altura) no pudo determinarse si se trató de un homicidio o un suicidio. Por su parte, la totalidad de casos tipificados como homicidios fueron producidos con armas blancas de fabricación casera o "cortes", lo que indica la incidencia del acceso 
a este tipo de elemento en los niveles de letalidad de los enfrentamientos entre internos. Por último, todas las muertes tipificadas como suicidios ocurrieron por ahorcamiento. ${ }^{19} \mathrm{Al}$ respecto, cabe resaltar las dificultades que presenta la tipificación de una muerte como suicidio, y más aún en el contexto penitenciario. Un estudio de Dooley (1990b) muestra que existe una importante discrepancia entre las muertes que pueden ser adjudicadas a un daño autoinfligido y las que acaban siendo tipificadas como suicidio. En particular, encuentra que generalmente son considerados como tales aquellos decesos que se parecen más a la típica imagen de suicidio (es decir, ocurren por la noche, sus víctimas son en su mayoría hombres y el método utilizado es el ahorcamiento).

Respecto a las causas de las muertes no violentas, el panorama es poco claro. Los registros médicos en este contexto son pobres en general y mucha información no se adjunta a las carpetas de los internos. Los dictámenes forenses no suelen adjuntarse tampoco y la información queda dispersa en los distintos servicios de salud. En el período estudiado, cuando por estas razones la causa no quedó del todo clara, las muertes fueron registradas en la categoría otras causas no violentas. En al menos 7 de las 19 muertes no violentas, se registró como causa una insuficiencia cardíaca, aunque no en todos los casos se verificaron afecciones previas. Tuvieron lugar también tres muertes por cáncer, una por un aneurisma, una por meningitis, una por una midriasis paralítica y una por congestión. ${ }^{20}$ En cuatro de las muertes que acabaron siendo catalogadas como naturales no se pudo recoger suficiente información sobre la causa, cuestión que indica con claridad las limitaciones de los registros médicos.

Tabla 3. Causas de muerte bajo custodia según tipo de muerte en 2017

\begin{tabular}{cccccc}
\hline \multicolumn{7}{c}{ Tipo de muerte } \\
\hline Causa de muerte & Homicidio & Suicidio & $\begin{array}{c}\text { Causa dudosa } \\
\text { [violenta) }\end{array}$ & $\begin{array}{c}\text { Enfermedad/ } \\
\text { Natural }\end{array}$ & Total \\
\hline Ahorcamiento & 0 & 10 & 0 & 0 & 10 \\
\hline $\begin{array}{c}\text { Herida de arma } \\
\text { blanca }\end{array}$ & 17 & 0 & 0 & 0 & 17 \\
\hline
\end{tabular}

19 No se detectaron en este período homicidios por ahorcamiento como simulación de suicidio, posibilidad desarrollada en el documento de la Procuración Penitenciaria de la Nación (2018, p. 373).

20 El hecho de que el formulario del Comisionado Parlamentario distinga solo entre VIH y otras enfermedades, y que todos los casos de muertes naturales ocurridos en 2017 correspondan a esta categoría residual, sugiere que sería pertinente afinar el sistema de categorías para registrar con mayor precisión los procesos que derivan en el fallecimiento por enfermedad dentro del sistema. 


\begin{tabular}{cccccc}
\hline & \multicolumn{5}{c}{ Tipo de muerte } \\
\hline Causa de muerte & Homicidio & Suicidio & $\begin{array}{c}\text { Causa dudosa } \\
\text { (violenta) }\end{array}$ & $\begin{array}{c}\text { Enfermedad/ } \\
\text { Natural }\end{array}$ & Total \\
\hline Caída de altura & 0 & 0 & 1 & 0 & 1 \\
\hline Muerte súbita & 0 & 0 & 0 & 1 & 1 \\
\hline $\begin{array}{c}\text { Otra enfermedad (no } \\
\text { VIH ni muerte súbita) }\end{array}$ & 0 & 0 & 0 & 11 & 11 \\
\hline $\begin{array}{c}\text { Otra causa no } \\
\text { violenta }\end{array}$ & 0 & 0 & 0 & 3 & 3 \\
\hline Sin datos & 0 & 0 & 0 & 4 & 4 \\
\hline Total & 17 & 10 & 1 & 19 & 47 \\
\hline
\end{tabular}

Fuente: Elaboración propia con base en datos del Comisionado Parlamentario para el Sistema Penitenciario, 2017.

Pasando a las explicaciones de los homicidios en prisión, el informe del Comisionado Parlamentario es claro en cuanto a que gran parte de los enfrentamientos que acaban en decesos fueron desatados a partir de hechos en apariencia "menores", que ponen de manifiesto las dificultades para sobrevivir en el contexto de la prisión. Entre ellos, destacan los conflictos relativos al acceso a plazas laborales (en particular aquellas que reportan posibilidades de movilidad en el espacio y contacto con otros), en un contexto en el cual priman el encierro y el ocio compulsivo. Otros elementos que se adicionan a esta problemática tienen que ver con la escasez de personal, los problemas de salud mental y el consumo problemático de drogas (que, a su vez, se vinculan a problemas de tráfico y endeudamiento, que potencian la situación de violencia reinante en ciertos módulos). Marcando una diferencia con lo que puede ocurrir con otros sistemas penitenciarios de la región, el informe del Comisionado Parlamentario pone de manifiesto que las muertes por homicidio ocurridas en las cárceles uruguayas responden, de un modo desproporcionado, a conflictos interpersonales resultantes de las pésimas condiciones de vida en los espacios de reclusión:

"[...] buena parte de los casos no se debieron a enfrentamientos entre bandas rivales, grupos o clanes sino a disputas extremas por bienes elementales como resultado de una convivencia signada por el encierro en celda y la falta de actividades sustantivas." (Comisionado Parlamentario para el Sistema Penitenciario, 2017, p. 22)

En cuanto a los suicidios, para el caso de Uruguay el Comisionado Parlamentario indica la presencia de múltiples señales de depresión o ideaciones suicidas los días u horas previos a las muertes. Sin embargo, y 
aun ante la presencia de síntomas claros, destaca la inexistencia o la escasez de respuestas institucionales que actúen como apoyo o contención. En este sentido, el contexto carcelario no solo dificulta la elaboración de planes o proyectos de vida a futuro, sino que también debilita fuertemente los apoyos afectivos y familiares de los internos, y actúa como un elemento clave en el agravamiento de eventuales disposiciones iniciales que pudieran presentar los individuos.

En todo caso, resulta evidente, a partir de los datos presentados, que el fenómeno de la muerte bajo custodia penitenciaria y sus distintas manifestaciones en Uruguay guardan un claro correlato con las condiciones de vida en el encierro en los distintos establecimientos. Al respecto, si bien el informe del Comisionado remarca la heterogeneidad del sistema, también sostiene que en algunos módulos o sectores de ciertos establecimientos se observa una "despresurización del Estado" (Comisionado Parlamentario para el Sistema Penitenciario, 2017, p. 35), en el sentido de que parece que hubiera desaparecido.

Específicamente, menciona que en esos lugares:

“[...] la convivencia es violenta y se presta para todo tipo de abusos y manipulaciones, amenazas, extorsiones. La desesperación por el bien más mínimo es causa de enfrentamiento. El clima es degradante. $\mathrm{Y}$ el contexto es un auténtico generador de violencia." (Comisionado Parlamentario para el Sistema Penitenciario, 2017, p. 16)

\section{Reflexiones finales}

Como ha quedado de manifiesto, el fenómeno de las muertes bajo custodia penitenciaria alcanza en Uruguay una magnitud preocupante. Esto ocurre en un contexto donde, a diferencia de lo observado en otros países de América Latina (como Brasil, por ejemplo), ${ }^{21}$ estas muertes no son producto de grandes matanzas o enfrentamientos entre bandas rivales (las cuales suelen tener gran repercusión en la opinión pública). En el caso de Uruguay, la muerte bajo custodia penitenciaria ocurre de modo sigiloso y dosificado, sin generar mayores alarmas en la ciudadanía y sin presentar mejoras significativas en los últimos años. Las posibilidades de morir dentro de las cárceles uruguayas (como resultado de la acción de terceros, de la propia persona o de procesos dudosamente catalogados de "naturales") son muy superiores a las que observamos en la vida extramuros. Por otro lado, dentro de las cárceles no solo se muere más, sino que se muere más joven.

Así, tanto la gravedad del tema como su importancia para el monitoreo de la violencia y de las posibilidades de supervivencia en las distintas

21 Para más información, ver el trabajo de Bogo Chies y Rotta Almeida (2019) en este mismo número. 
unidades penitenciarias hacen que sea vital su incorporación a los sistemas de información pública. El vacío de datos respecto de la muerte bajo custodia en Uruguay se suma a una necesidad aún más profunda y general, en tanto no existe un sistema de producción y difusión de estadísticas públicas básicas sobre la realidad del sistema penitenciario. La conformación de un sistema penitenciario nacional y los avances en la informatización de los registros observados en los últimos años son elementos que dan cuenta de un contexto propicio para comenzar a pensar en la elaboración de indicadores válidos y confiables, que no solo permitan visibilizar la muerte bajo custodia, sino también otros aspectos relativos a la calidad de vida en las prisiones y a los niveles de violencia dentro de ellas (por ejemplo, el número de heridos en enfrentamientos o de intentos de autoeliminación).

En este sentido, se requiere información detallada y de calidad sobre los eventos que desembocan en fallecimientos y sobre los perfiles e historias vitales de las personas privadas de libertad, así como sobre las condiciones de vida en el encierro, para poder contrastar de manera empírica las hipótesis derivadas de las principales teorías que buscan explicar la violencia en las cárceles (la teoría de la importación y la de la deprivación, respectivamente). Solo así se podrá analizar el grado en que ambos tipos de factores explicativos se encuentran presentes en la realidad carcelaria uruguaya y en qué medida interactúan y se retroalimentan. Además, la posibilidad de generar información empírica en contextos de encierro caracterizados por la presencia de carencias críticas, como los latinoamericanos, posiblemente resulte en un refinamiento conceptual y explicativo, en la medida en que aporte información valiosa a un debate teórico desarrollado sobre todo en países anglosajones, donde existe un piso más consolidado de condiciones mínimas y de respeto de derechos básicos dentro de las prisiones. Por su parte, la generación de información sistemática también tendrá consecuencias positivas desde el punto de vista social, en la medida en que brinde claves para eventuales intervenciones que permitan reducir la cantidad de muertes, particularmente en las cárceles que tienen los mayores niveles de morbilidad.

Por otro lado, y más allá de los avances que se deberían observar desde el propio sistema penitenciario en la disponibilidad y la calidad de la información relevada, uno de los desafíos para el monitoreo de las muertes en custodia es la posibilidad de que los organismos responsables de él no dependan del Instituto Nacional de Rehabilitación o del Ministerio del Interior, en especial para el registro de variables sensibles, como la categorización del tipo de muerte (Gual, 2016, p. 45). Este cambio demandaría el esfuerzo conjunto de los diversos actores implicados (organizaciones de la sociedad civil, academia y organismos gubernamentales) a los efectos de lograr información de calidad.

En particular, la categorización de los distintos tipos de muerte presenta al menos dos desafíos. Por un lado, es necesario avanzar en la construcción 
de tipologías que sean lo suficientemente sensibles a distintos aspectos clave de los procesos que desembocan en una muerte, de modo tal de dar cuenta de posibles omisiones sociales y estatales en la generación de condiciones de reclusión respetuosas de los derechos humanos. Por otro lado, resulta necesario avanzar en la coordinación y el intercambio regional respecto a los distintos dispositivos de categorización, a los efectos de lograr información comparable de mayor calidad y escala, que permita analizar tendencias regionales en el tiempo.

Para finalizar, deseamos dejar claro que, si bien es muy probable que tanto los elementos importados por los individuos como los derivados de las condiciones de encierro interactúen en la explicación de la mayoría de las muertes ocurridas en el sistema, entendemos, con Liebling (2017), que el hecho de que existan factores de importación o de riesgo individual no exime al Estado de su responsabilidad, e incluso la refuerza. Entendemos que cada uno de los tipos de muerte representa una omisión específica del Estado en garantizar el derecho a la vida de los internos, problema que se vuelve particularmente evidente en algunos establecimientos (o incluso módulos dentro de las unidades más grandes), donde la muerte bajo custodia penitenciaria ha pasado a ser un fenómeno más o menos habitual. En aquellas unidades que tienen más volumen de personas privadas de libertad, menos proporción de funcionarios por interno, menos acceso a actividades laborales, educativas y recreativas, así como peores condiciones de vida, la muerte - en sus distintas manifestaciones- se torna un evento "esperable", más allá de los rasgos de las personas que se encuentran alojadas en ellas. 


\section{Referencias bibliográficas}

Aebi, M. F.; M. M. Tiago; L. Berger-Kolopp y C. Burkhardt (2017). SPACE I-Council of Europe Annual Penal Statistics: Prison populations. Survey 2016. Estrasburgo: Council of Europe.

Arbesún, R. (2017). La “imposible prisión”: una historia de la reforma penitenciaria uruguaya [en línea]. Disponible en: $<\mathrm{https}$ :/www.prisoninsider.com/files/d8746405/la_imposible_prision_uruguay_cp_4.pdf $>$ [acceso: 22/05/2019].

Bogo Chies, L. A. y Rotta Almeida, B. (2019). Mortes sob custodia prisional no Brasil. Prisões que matam; mortes que pouco importam. Revista de Ciencias Sociales, 32(45), pp. 67-90.

Comisionado Parlamentario para el Sistema Penitenciario (2016). Informe especial sobre muertes en prisión en 2016 [en línea]. Disponible en: $<$ https://parlamento.gub.uy/cpp/documentos/informes-al-parlamento $>$ [acceso: 22/05/2019].

Comisionado Parlamentario para el Sistema Penitenciario (2017). Boletín estadístico 2016 [en línea]. Disponible en: <https://parlamento.gub.uy/cpp> [acceso: 22/05/2019].

Comisionado Parlamentario para el Sistema Penitenciario (2018a). Informe anual 2017 [en línea]. Disponible en: <https://parlamento.gub.uy/cpp> [acceso: 22/05/2019].

Comisionado Parlamentario para el Sistema Penitenciario (2018b). Boletín estadístico 2017 [en línea]. Disponible en: <https://parlamento.gub.uy/cpp> [acceso: 22/05/2019].

Dawes, J. (1999). Deaths in custody: moving beyond a statistical analysis. En 3rd National Outlook Symposium on Crime in Australia. Mapping the Boundaries of Australia's Criminal Justice System, Instituto Australiano de Criminología, Canberra.

Dooley, E. (1990a). Prison suicide in England and Wales, 1972-87. British Journal of Psychiatry, 156(01), pp. 40-45.

Dooley, E. (1990b). Unnatural deaths in prison. British Journal of Criminology, 30(2), pp. 229-234.

Goffman, E. (2001[1961]). Internados. Ensayos sobre la situación social de los enfermos mentales. Buenos Aires: Amorrortu Editores. 
Gual, R. (2016). La muerte bajo custodia penal como objeto de investigación social: una perspectiva regional. Dossiê Punição e controle social: degradações carcerárias em América Latina e Europa, 2(2), pp. 29-48.

Gual, R. (2019). La prisión irresistible. Muertes por autoagresión bajo custodia penitenciaria en argentina. Revista de Ciencias Sociales, 32(45), pp. 91-118.

Instituto Nacional de Estadística (2018). Indicadores demográficos [en línea]. Disponible en: <http://www.ine.gub.uy/web/guest/indicadoresdemograficos $1>$ [acces: 22/05/2019].

International Centre for Prison Studies (2018). World Prison Brief [en línea]. Disponible en: <http://www.prisonstudies.org/country/uruguay $>$ [acceso: 22/05/2019].

Irwin, J. K. y D. R Cressey (1962). Thieves, convicts and the inmate culture. Social Problems, 10(2), pp. 142-155.

Levcovitz, E.; M. Fernández Galeano; R. Rodríguez Buño y W. Benia (coords.) (2016). Salud y enfermedad en condiciones de privación de libertad: diagnóstico epidemiológico. Montevideo: OPP.

Liebling, A. (1999). Prison suicide and prisoner coping. Crime and Justice, 26, pp. 283-359.

Liebling, A. (2011). Moral performance, inhuman and degrading treatment and prison pain. Punishment and Society, 13(5), pp. 530-550.

Liebling, A. (2017). The meaning of ending life in prison. Journal of Correctional Health Care, 23(1), pp. 20-31.

Mecanismo Nacional de Prevención de la Tortura-Paraguay (2017). Muertes bajo custodia. Registro nacional de personas fallecidas en instituciones de privación de libertad 2013-2016. Asunción: Mecanismo Nacional de Prevención de la Tortura-Paraguay.

Mecanismo Nacional de Prevención de la Tortura-Uruguay (2017). Análisis de contexto y monitoreo del sistema carcelario de adultos y unidades policiales [en línea]. Disponible en: <https://www.gub.uy/institucionnacional-derechos-humanos-uruguay/comunicacion/publicaciones/ informe-anual-del-mnp-2017-sistema-carcelario-de-adultos-y-unidades $>$ [acceso: 22/05/2019]. 
Ministerio del Interior (2018). Evolución de los homicidios en Uruguay [en línea]. Disponible en: <https://www.minterior.gub.uy/index.php/ unicom/noticias/5534-evolucion-de-los-homicidios-en-uruguay> [acceso: 22/05/2019].

Ministerio de Salud Pública, División Estadísticas Vitales (2017). "Cuadro 6. Indicadores varios" [en línea]. Disponible en: http://estadisticas.msp.gub. uy/index.php/home [acceso: 22/05/2019].

Parlamento del Uruguay (2018). Comisionado informa al Fiscal y al Parlamento sobre muerte en el Penal de Libertad. Parlamento del Uruguay [en línea]. Disponible en: <https://parlamento.gub.uy/cpp/actividades/noticias/89819> [acceso 13/02/2019].

Programa de Naciones Unidas para el Desarrollo-Uruguay (2018). Uruguay en la actualización estadística del IDH 2018 [en línea]. Disponible en: < http:// www.uy.undp.org/content/uruguay/es/home/presscenter/articles/2018/09/ Uruguay-actualizacion-IDH-2018.html $>$ [acceso: 22/05/2019].

Procuración Penitenciaria de la Nación (2016). Informe estadístico sobre muertes en prisión. Buenos Aires: Equipo de fallecimientos en prisión, Observatorio de Cárceles Federales, Procuraduría Penitenciaria de la Nación.

Procuración Penitenciaria de la Nación (2018). Informe anual 2017: la situación de los derechos humanos en las cárceles federales de la Argentina. Buenos Aires: Procuración Penitenciaria de la Nación.

Rossi, J. P. (2017). Salud mental y privación de libertad: suicidios en cárceles. Apuntes sobre el trabajo realizado desde la Oficina del Comisionado Parlamentario Penitenciario. En P. Hein y C. Larrobla (coords.). 70 años de suicidio en Uruguay: 7 disciplinas, 7 entrevistas, 7 encuentros. Montevideo: CSIC, UdelaR, pp. 97-106.

Sánchez, A. (2016). Muerte en custodia. Revista Virtual Intercambios [en línea], 17. Disponible en: <http://intercambios.jursoc.unlp.edu.ar/> [acceso: 22/05/2019].

Sosa Barón, S. (2018). Muerte natural: alcances y limitaciones del concepto como dispositivo de desciframiento de las muertes en custodia. Revista Epistemología e Historia de la Ciencia, 3(1), pp. 61-74.

Sykes, G. (1958). The society of captives: a study of a maximum security prison. Nueva Jersey: Princeton University Press. 
Talvi, E. (coord.) (2017). Privación de libertad y reinserción social en Uruguay. Montevideo: CERES-CAF.

Trajtenberg, N. y O. Sánchez de Ribera (2019). Violencia en instituciones penitenciarias. Definición, medición y explicación del fenómeno. Revista de Ciencias Sociales, 32(45), pp. 147-175.

\section{Contribución de autoría}

Ana Vigna contribuyó en un 51\% a la redacción de este artículo, mientras que Santiago Sosa lo hizo en un $49 \%$. 